\title{
Energy Saving Efficiency Comparison of Transmit Power Control and Link Adaptation in BANs
}

\author{
Qi Zhang \\ Aarhus University, Aarhus, Denmark. \\ Email: qz@iha.dk
}

\begin{abstract}
The wireless channels in Body Area Networks (BANs) have significant temporal variations due to body movements. Therefore there is a potential to save energy by exploiting adaptive schemes, such as transmit power control (PC) and link adaptation (LA). This paper investigates the energy saving efficiency of PC and LA in BANs. The theoretical bounds of energy saving efficiency of PC and LA, and the condition that LA outperforms PC are derived. Generally speaking, LA is more efficient in saving energy than PC in BANs. The energy saving efficiency and packet erasure rate (PER) of PC and LA are evaluated through simulations with a large measured dataset from a BAN testbed. The simulation results show that LA can save $80-85 \%$ more energy than PC during $80 \%$ time. The increased PER due to the adaptive schemes is below $1 \%$ during $80-95 \%$ time at the different links.
\end{abstract}

\section{INTRODUCTION}

Wireless Body Area Network (BAN) is an emerging technology to continuously collect physiological data for diagnosis or deliver various personalized therapeutic treatment related applications and services. The sensor nodes in BANs are required to be small and lightweight to be able to deliver the level of comfort and unobtrusiveness [1]. This requirement sets the limitation on the battery size and makes energy efficiency even more crucial. The common goal of various energy efficient solutions is to make sensor nodes adapt energy consumption in response to the changes in the environment, by which to minimize energy usage. All layers in the system from transceiver architecture to network protocol and algorithm can exploit energy efficient design; nevertheless, it is critical that energy efficient protocol and algorithm is based on accurate modeling of the underlying hardware [2]. The wireless channels in BANs have significant temporal variations due to body movements [1], [3], [4]. The fluctuation of the path loss can reach $\pm 30 \mathrm{~dB}$ around the median [1]. Therefore, there is a potential in energy saving by using adaptive schemes. Transmit power control (PC) and link adaptation (LA) are the two possible adaptive schemes widely used for the dynamic wireless channels. Transmit power control have been used to save energy in BANs [5]-[7]. Rate adaptation used to save energy in WSNs has been addressed in [8].

This paper evaluates PC and LA schemes by considering the overall energy consumption at the transmitter and receiver, including both RF transmission and transceiver circuit energy consumption. It is aiming to answer the following questions. What impacts the theoretical bound of energy saving in PC and LA, respectively? How much energy saving can be achieved by the adaptive schemes in the real BAN links?

The theoretical analysis shows that in BANs PC is not efficient in saving energy as in short-range communication systems the RF transmit power is much smaller than the power consumption of the transceiver circuit. The energy saving efficiency of PC depends on the average current at the transmitter and receiver. For a given transceiver the maximum energy saving efficiency of PC is bounded by the current at the receiver and the minimum current at the transmitter. The energy saving efficiency of LA is monotonically increases when the average data rate increases. LA is more efficient in saving energy than PC, if it meets the condition that the average data rate exceeds a threshold which is determined by the average current at the transmitter and receiver in PC. According to the data sheets of the transceivers off-theshelf, this condition holds. The performance of PC and LA is evaluated by a simulation with a large dataset collected from an open-source BAN testbed [9]. The simulation results show that LA is more energy efficient than PC. LA can save about $80-85 \%$ more energy during $80 \%$ time and in the worst case $50 \%$ more energy can be saved. Furthermore, the implemented LA and PC schemes have similar packet erasure rate (PER). Comparing with the static scheme the increased PER of PC is below $1 \%$ during $80-95 \%$ time for different links.

This paper is organized as follows. The related work is presented in Section II. Section III and IV describe transmit power control and link adaptation schemes, respectively. Section V theoretically compares LA and PC in energy saving efficiency and derives the theoretical bounds and condition. The performance of LA and PC are evaluated through simulation in Section VI. The conclusion is reached in Section VII.

\section{RELATED WORK}

Many researchers have worked on dynamic transmit power control as a means to save energy in wearable BANs, such as [5]-[7]. S. Xiao et al. [5] proposed a dynamic PC scheme which can adapt transmit power by comparing the running RSSI average to the pre-defined lower and upper threshold. The theoretical benefit of adaptive transmit PC can save nearly $35 \%$ energy without compromising reliability across different scenarios in [5]. In practice, comparing to the case of using maximum transmit power it can save $14-30 \%$ energy in exchange for 1-10\% packet losses. M. Quwaider et al. [6] developed several customized approaches for transmit $\mathrm{PC}$ via tracking of postural node mobility. It evaluated the 
performance based on Mica2Mote using radio chip CC1000. With one of the proposed PC schemes, DPPI, it can save 43$50 \%$ energy for different testing persons, comparing to using the maximum transmit power. D. Smith et al. [7] proposed a novel channel prediction which can effectively predict the channel state in BANs up to $2 \mathrm{~s}$ ahead. The dynamic transmit $\mathrm{PC}$ using the novel predictor can both reduce transmit power and improve reliability. It declared that $8-22 \%$ energy was saved comparing with a constant transmit power of $10 \mathrm{dBm}$.

A. Wang et al. has explained in [10] that data rate does not scale with power consumption due to the fixed power consumption cost of the transmitter electronics. According to [11], the power consumption of transceiver circuit electronics is fixed when the data rates are below $10 \mathrm{Mbps}^{1}$. This conclusion indicates that increasing data rate can save energy on transmission and receiving due to the shortened transmission and receiving time. [11], [12] also addressed that the power consumption is dominated by the circuit electronics (e.g., frequency synthesizer, mixer, etc.) for short-range RF transceivers operating in the Giga-Hertz carrier frequency range. Furthermore, [12] has shown that M-ary modulation may enable energy savings over binary modulation for some short-range applications by reducing the transmission and receiving time.

S. Lanzisera et al. [8] have looked into using data rate adaptation to save energy in wireless sensor networks. It inferred the link quality based on RSSI or LQI measurement. Then based on the link quality it realized rate adaptation through adapting the spreading factor of direct sequence spread spectrum used in 802.15 .4. It stated that about $40 \%$ average energy saving was achieved in their testbed. F. Martelli et al. [13] proposed to use link adaption by selecting different modulation schemes aiming to reduce packet losses in BANs.

\section{TRANSMit POWER CONTROL}

The basic idea of transmit PC is to optimally select an RF transmit power to achieve the required performance (e.g., network capacity, reliability, energy consumption etc.) at a constant data rate. Transmit PC is often used as a means to avoid near-far effect or a means of interference mitigation in interference limited wireless communication system, such as WCDMA cellular networks. PC has also been used in mobile handset in cellular networks to save energy. Cellular network is a typical long-range communication system (i.e., transmission distance $\geq 100 \mathrm{~m}$ ) and power consumption of power amplifier (PA) dominates the transceiver power consumption at handset. The power consumption of PA, $P_{P A}$, can be simply represented by RF transmit power, $P_{\text {out }}$, and efficiency of PA, $\eta_{P A}$, as $P_{P A}=P_{\text {out }} / \eta_{P A}$.

The transceiver energy efficiency, $\eta$, is defined by [11].

$$
\eta=\frac{P_{\text {out }}}{P_{P A}+P_{c i c}^{t x}+P_{c i c}^{r x}}
$$

\footnotetext{
${ }^{1}$ The power consumption of the circuit blocks will increase with data rate when the data rates beyond a few 10's Mega-bits per second [11].
}

Where, $P_{c i c}^{t x}, P_{c i c}^{r x}$ are the power consumption of circuit block in transmitter and receiver, respectively.

When PA dominates the transceiver power consumption (e.g., long-range communication systems), $P_{c i c}^{t x}+P_{c i c}^{r x}$ is very small comparing to $P_{P A}$. Hence, $\eta$ can be approximated as [11]

$$
\eta \approx \frac{P_{\text {out }}}{P_{P A}}=\eta_{P A}
$$

Efficiency of PA, $\eta_{P A}$, can vary quite a bit depending on PA type, RF transmit power, technology and design. Normally $\eta_{P A}$ decreases quickly with decreasing output power [8], nevertheless, for simplicity a fixed efficiency of PA, $\eta_{P A}$, is assumed here. Then it is clear that the energy consumption will approximately scale linearly with the RF transmit power. Therefore, in a system that PA power consumption is dominant, $\mathrm{PC}$ can be quite efficient in saving energy. For example, ideally reducing $P_{\text {out }}$ by $3 \mathrm{~dB}$ can approximately reduce half of the energy consumption at the transmitter. If considering the total energy consumption at both transmitter and receiver, the energy saving will be less than $50 \%$, because PC cannot save energy at the receiver.

However, in low power short-range communication system (i.e., transmission distance $<10 \mathrm{~m}$ ), such as BANs and WSNs, the power consumption of transceiver electronics dominates. $P_{P A}$ is about an order of magnitude less than the power consumption of transceiver electronics. Thus transceiver energy efficiency $\eta$ can be approximated as [11]

$$
\eta \approx \frac{P_{\text {out }}}{P_{c i c}^{t x}+P_{c i c}^{r x}}
$$

We can see that transceiver energy efficiency $\eta$ goes down when reducing the RF transmit power. In such a system the energy consumption does not scale linearly with the RF transmit power; therefore, $\mathrm{PC}$ is not efficient in energy saving. Taking a widely used radio chip in WSNs, CC2420 [14], as an example, when reducing $P_{\text {out }}$ from $0 \mathrm{dBm}$ to $-3 \mathrm{dBm}$, it can reduce the transmitter energy consumption by only $12.79 \%$ not $50 \%$. If taking the energy cost at the receiver into account, the energy saving is only about $6 \%$.

\section{LINK ADAPTATION}

The basic idea of link adaptation (LA) scheme is to adapt data rate by selecting a suitable modulation and coding scheme at a constant transmit power. The goal of LA is to transmit a certain amount of data using minimum resource at an acceptable reliability. The resource could be energy, time, frequency spectrum, and many others. LA is suitable to be used in an environment with dynamic path loss and channel quality. The reason is as follows. In a static scheme, the data rate and transmit power are fixed. In order to work properly in the worst case a static scheme usually has to transmit at the lowest data rate. The problem of the static scheme is obvious that it misses the opportunities of transmitting at a higher data rate when the channel is good. Comparing with the static scheme, LA has a potential to save energy, reduce channel occupation time, improve spectrum efficiency and others. We 
focus on energy saving in this paper as energy is one of the crucial challenges in BANs.

When the transmit and receiving power is fixed, the energy consumption scales linearly with time. The saved energy by LA results from the reduced transmission and receiving time at the transmitter and receiver. Hence, the saved energy scales linearly with the reduced transmission and receiving time. For example, by changing spreading factor from 4 to 2, it can save approximately $50 \%$ energy.

\section{Link AdAptation vs. Power Control}

To compare the energy saving efficiency of LA and PC, a generic energy model is used. In this model the transition time between sleep and active is not considered. If the transition time is longer than the active duration, LA cannot give too much payoff. However, if a transceiver has a long transition time, a short active duration should be avoided in scheduling design.

TABLE I

DenOtations USED IN SECTION V

\begin{tabular}{|c|c|c|}
\hline Notation & Meaning & Unit \\
\hline$P_{t x}$ & overall power consumption at transmitter & $\mathrm{mW}$ \\
$P_{r x}$ & overall power consumption at receiver & $\mathrm{mW}$ \\
$t$ & transmission (receiving) time & $\mathrm{s}$ \\
$V$ & voltage & volt \\
$I_{0}$ & current at maximum transmit power $=0 \mathrm{dBm}$ & $\mathrm{mA}$ \\
$I_{r}$ & current for receiving & $\mathrm{mA}$ \\
$R_{0}$ & minimum data rate & $\mathrm{kbps}$ \\
$I_{i}$ & current at transmit power setting $i$ & $\mathrm{~mA}$ \\
$I_{i}$ & mean current at transmitter with PC & $\mathrm{mA}$ \\
$R_{j}$ & data rate setting $j$ & $\mathrm{kbps}$ \\
$R_{j}$ & mean data rate with LA & $\mathrm{kbps}$ \\
$E_{p c}$ & total energy consumption with PC & $\mathrm{mJ}$ \\
$E_{l a}$ & total energy consumption with LA & $\mathrm{mJ}$ \\
$E_{0}$ & total energy consumption with static scheme & $\mathrm{mJ}$ \\
$L$ & packet size & $\mathrm{bit}$ \\
$N$ & total number of packets & \\
$\xi_{p c}$ & energy saving efficiency with PC & \\
$\xi_{l a}$ & energy saving efficiency with LA & \\
$\xi$ & energy saving efficiency of LA over PC & \\
\hline \multicolumn{2}{|}{} \\
\hline
\end{tabular}

The basic static scheme is assumed to use a constant transmit power at a fixed data rate (i.e., the maximum transmit power and the minimum data rate). Assuming no packet loss, the total energy consumption of the static scheme used to transmit $N$ packets from the sender to the receiver, $E_{0}$, basically includes the energy spent on transmission and receiving, respectively. It can be calculated as

$$
\begin{aligned}
E_{0} & =\left(P_{t x}+P_{r x}\right) t \\
& =V\left(I_{0}+I_{r}\right) \frac{N L}{R_{0}}
\end{aligned}
$$

Where, $P_{t x}=P_{P A}+P_{c i c}^{t x}$ and $P_{r x}=P_{c i c}^{r x}$. Assume $I_{r}=\gamma I_{0}$,

$$
E_{0}=(1+\gamma) \frac{V I_{0} N L}{R_{0}}
$$

With PC, the current at the transmitter varies based on the different transmit power, which results in the variations of the power consumption at the transmitter. Nevertheless, PC has no impact on the power consumption at the receiver. Thus, the energy used to transmit $N$ packets from the sender to the receiver with $\mathrm{PC}$ scheme, $E_{p c}$, can be expressed as

$$
E_{p c}=V \sum_{n=1}^{N}\left(I_{i}+I_{r}\right) \frac{L}{R_{0}}
$$

Assume the mean current over the transmission of the $N$ packets $\overline{I_{i}}=\alpha I_{0}$, where $\alpha \leq 1$. Then there is

$$
\begin{aligned}
E_{p c} & =V\left(\alpha I_{0}+\gamma I_{0}\right) \frac{N L}{R_{0}} \\
& =(\alpha+\gamma) \frac{V I_{0} N L}{R_{0}}
\end{aligned}
$$

With LA, the transmission and receiving time varies based on the different data rate. Traditionally a change in modulation, for example, from a basic BPSK to M-PSK, would cause an increase in power consumption of transceiver electronics due to the fact that a quadrature modulator would have to be employed in order to support the inherent increase in phase resolution. [12] also tried to model the increased power in M-ary modulation on the frequency synthesizer and other circuit. However, in the case of an IEEE 802.15.6 compliant transmitter this is not the case, because the defined binary modulation scheme consists of a $\pi / 2$ rotated version of a DBPSK [15]. The rotation is only possible by employing the quadrature modulator circuitry. Therefore, power consumption will not significantly increase in the transceiver electronics by switching from $\pi / 2$ DBPSK to $\pi / 2$ DQPSK. The power consumption at the transmitter and receiver is assumed to be constant here. Thus, the total energy used to transmit $N$ packets from the sender to the receiver with LA can be expressed as

$$
E_{l a}=V\left(I_{0}+I_{r}\right) \sum_{n=1}^{N} \frac{L}{R_{j}}
$$

Assume the mean data rate over the transmission of the $N$ packets $\overline{R_{j}}=\beta R_{0}$, where $\beta \geq 1$. Then there is

$$
\begin{aligned}
E_{l a} & =V\left(I_{0}+\gamma I_{0}\right) \frac{N L}{\beta R_{0}} \\
& =\frac{1+\gamma}{\beta} \cdot \frac{V I_{0} N L}{R_{0}}
\end{aligned}
$$

Comparing with the static scheme, the energy saving efficiency with transmit PC can be calculated as follows.

$$
\begin{aligned}
\xi_{p c} & =\left(E_{0}-E_{p c}\right) / E_{0} \\
& =\frac{1-\alpha}{1+\gamma}
\end{aligned}
$$

From Equation 1, it shows that the energy saving efficiency of PC depends on the average current at the transmitter and the current at the receiver. The maximum energy saving efficiency of PC is bounded by the current at the receiver and the minimum current at the transmitter, i.e., $\xi_{p c} \leq \frac{1-\alpha_{\min }}{1+\gamma}$. For example, as we know in BANs, the power consumption of transceiver electronics dominates. The current at a receiver could be very high and even higher than the maximum current 
at a transmitter. Hence, the energy saving efficiency of PC could be quite low. This is consistent with the analysis in Section III.

Comparing with the static scheme, the energy saving efficiency of LA, $\xi_{l a}$, can be calculated in the following.

$$
\xi_{l a}=\left(E_{0}-E_{l a}\right) / E_{0}=1-\frac{1}{\beta}
$$

Equation 2 shows that the energy saving efficiency of LA only depends on the average data rate, i.e., $\xi_{l a}$ monotonically increases when the average data rate increases.

To tell whether LA is more efficient than PC in saving energy, we can compare $E_{p c}$ and $E_{l a}$ as follows.

$$
\begin{aligned}
\xi & =\left(E_{p c}-E_{l a}\right) / E_{p c} \\
& =1-\frac{1+\gamma}{\beta(\alpha+\gamma)}
\end{aligned}
$$

Equation 3 shows that as long as $\beta>\frac{1+\gamma}{\alpha+\gamma}$ link adaptation will outperform power control in saving energy.

\section{Vi. Performance Analysis}

\section{A. Numerical Analysis}

In this subsection, we will look at the limit of the energy saving efficiency with PC for the radio chips off-the-shelf. Table II lists some relevant data of radio chips: CC2420 [14], CC1000 [16], CC2520 [17] which are often used in WSNs. $\Delta_{\text {out }}$ is the difference of the minimum transmit power comparing to $0 \mathrm{dBm}$. From Table II we can see that the transceiver energy efficiency is very low in these transceivers for shortrange communications. Based on the data, we obtain the maximum energy saving efficiency of $\mathrm{PC}, \xi_{p c}^{\max }$, for different transceivers, which is in the range of $(0.1970,0.3028)$.

TABLE II

$I_{0}, I_{m i n}, I_{r}$ OF DIFFERENT RATIO CHIPS

\begin{tabular}{|c|c|c|c|c|}
\hline Radio Chip & CC2420 & CC1000 & CC1000 & CC2520 \\
\hline Frequency (MHz) & 2400 & 433 & 868 & 2400 \\
$I_{0}(\mathrm{~mA})$ & 17.4 & 10.4 & 16.5 & 25.8 \\
$\eta_{\max }$ & $0.92 \%$ & $1.87 \%$ & $1.28 \%$ & $0.75 \%$ \\
$I_{\min }(\mathrm{mA})$ & 8.5 & 6.9 & 8.6 & 16.2 \\
$\Delta_{\text {out }}(\mathrm{dB})$ & 25 & 20 & 20 & 18 \\
$I_{r}(\mathrm{~mA})$ & 18.8 & 7.4 & 9.6 & 18.5 \\
$\alpha_{\min }$ & 0.489 & 0.663 & 0.521 & 0.628 \\
$\gamma$ & 1.08 & 0.711 & 0.582 & 0.717 \\
$\xi_{\text {pax }}^{\text {max }}$ & 0.2457 & 0.1970 & 0.3028 & 0.2167 \\
\hline
\end{tabular}

Generally, the receiver sensitivity is assumed to approximately deteriorate $3 \mathrm{~dB}$ when the data rate is doubled. For example, by decreasing spreading factor by half, the data rate is doubled but $3 \mathrm{~dB}$ processing gain is lost. Or by switching from BPSK to QPSK, the energy per symbol to noise density ratio $E_{s} / N_{0}$ increases $3 \mathrm{~dB}$. The maximum data rate is assumed to be eight times of the minimum data rate in LA, although LA is not widely supported by the transceivers off-the-shelf. Based on these assumptions, when the path loss is reduced by $3 \mathrm{~dB}$, with transmit PC ideally it can reduce the transmit power by $3 \mathrm{~dB}$ or alternatively it can double the data rate with $\mathrm{LA}$.
We denote $\beta^{\text {th }}=\frac{1+\gamma}{\alpha+\gamma}$ the threshold of $\beta$ that LA is more efficient than PC in saving energy. Table III lists $\beta^{\text {th }}$ for CC2420 and CC2520 as example. It is clear that the condition $\beta \geq \beta^{\text {th }}$ always holds.

TABLE III

THRESHOLD OF LINK ADAPTATION OUTPERFORMING POWER CONTROL IN CC2420 AND CC2520.

\begin{tabular}{|c|c|c|c|c|c|}
\hline$P_{\text {out }}(\mathrm{mW})$ & $\alpha_{C C 2420}$ & $\beta_{C C 2420}^{t h}$ & $\alpha_{C C 2520}$ & $\beta_{C C 2520}^{t h}$ & $\beta$ \\
\hline 1 & 1 & 1 & 1 & 1 & 1 \\
0.5 & 0.8736 & 1.0647 & 0.9259 & 1.0451 & 2 \\
0.25 & 0.7529 & 1.1348 & 0.8028 & 1.1298 & 4 \\
0.125 & 0.6624 & 1.1938 & 0.7131 & 1.2006 & 8 \\
\hline
\end{tabular}

\section{B. Simulation Results}

To evaluate the energy saving efficiency of LA and PC in BANs, we apply the two adaptive schemes to a large dataset in the simulation. The dataset was continuously sampled at 200 $\mathrm{Hz}$ over 12 hours with transmit power $0 \mathrm{dBm}$ in a wearable BAN testbed at NICTA [9], [18], [19]. A simple LA algorithm and a PC algorithm were implemented in the simulation. Perfect feedback is assumed in the simulation, which just provides an equal condition for comparision of LA and PC. The basic idea of LA is that if the RSSI at the receiver is higher than the current receiver sensitivity, then it selects a suitable data rate setting for the next transmission and updates the receiver sensitivity based on the selected data rate. If the RSSI is below the current receiver sensitivity, then the packet is regarded as lost. A lower data rate should be selected for the next packet transmission. Regarding how to adapt data rate, there could be many options. A step-wise increase and fast decrease algorithm is implemented in the simulation. Note that in the simulation the packet erasures in LA are the original packet erasures in the real measurement plus those below the receiver sensitivity. The data rate options for LA are based on what is defined in IEEE 802.15.6 standard [15] (see Table IV).

TABLE IV

DATA RATE DEFINED IN IEEE 802.15.6 AT 2400-2483.5 MHz [15]

\begin{tabular}{|l|l|l|l|l|l|}
\hline$R_{j}$ & Modulation & $\begin{array}{l}\text { Spreading } \\
\text { Factor }\end{array}$ & $\begin{array}{l}\text { Data Rate } \\
(\mathrm{kbps})\end{array}$ & $\begin{array}{l}\text { Receiver Sensitivity } \\
(\mathrm{dBm})\end{array}$ \\
\hline$R_{1}$ & $\pi / 2$ DBPSK & 4 & 121.4 & -92 & \\
$R_{2}$ & $\pi / 2$ DBPSK & 2 & 242.9 & -90 & \\
$R_{3}$ & $\pi / 2$ DBPSK & 1 & 485.7 & -87 & \\
$R_{4}$ & $\pi / 2$ DQPSK & 1 & 971.4 & -83 & \\
\hline
\end{tabular}

The basic idea of the PC algorithm is that if the current transmit power minus the path loss is higher than the basic receiver sensitivity $-92 \mathrm{dBm}$, then it calculates a new transmit power and sends the next packet at the new transmit power. If the current transmit power minus the path loss is below -92 $\mathrm{dBm}$, the packet is regarded as lost. There could be different strategies to adapt the transmit power. Similarly to LA, a step-wise decrease and fast increase strategy is used in the simulation. To make a comparison with LA in energy saving, the $\mathrm{PC}$ algorithm can select one of the transmit power in the set of $[0,-2,-5,-9] \mathrm{dBm}$ which is derived based on the receiver sensitivity given in Table IV. 


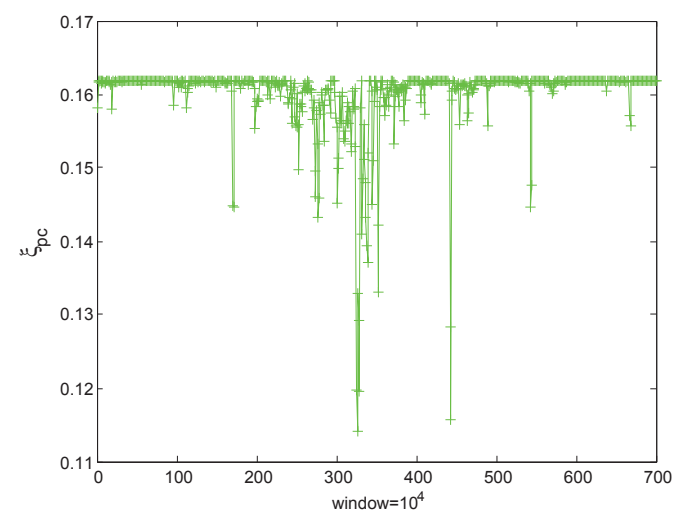

(a) The link of the right hip $\rightarrow$ the right ankle.

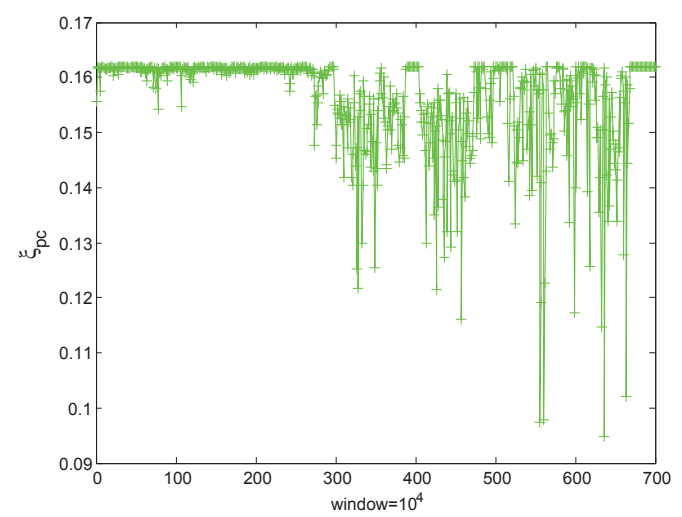

(b) The link of the right hip $\rightarrow$ the right wrist.

Fig. 1. The energy saving efficiency of power control.

In the simulation, the voltage and current data is taken from the data sheet of radio chip CC2420 [14]. Each value is averaged over $10^{4}$ samples (i.e., window $=10^{4}$ ). Figure $1(\mathrm{a})$ and 1 (b) show the energy saving efficiency of PC for the links from the right hip to the right ankle and from right hip to the right wrist, respectively. We can see that comparing with the static scheme, PC can at most save approximately $16 \%$ energy. The energy saving efficiency can drop below $10 \%$ in the worst case. Figure 2(a) and Figure 2(b) show how many percent that LA is more efficient than PC. We can see that similarly to Figure 1 the energy saving efficiency in Figure 2 varies over time owing to the time-variant channel characteristics in BAN. In the links to the right ankle and to the right wrist, the LA can save $80-85 \%$ more energy than PC during $80 \%$ time. And in the worst case, LA can still save about $50 \%$ energy more than PC, in other words, in the worst case LA only uses half of the energy used by PC.

Figure 3(a) and Figure 3(b) show the packet erasure rate (PER) with LA and PC at the two links. We can see that with the simple LA and PC algorithms, the PER of these two adaptive schemes are almost same. Generally the PER is below 5\%. There are a few burst losses in which the PER can reach $20-25 \%$ which includes the original packet losses with static scheme. Hence, it is necessary to see the increased

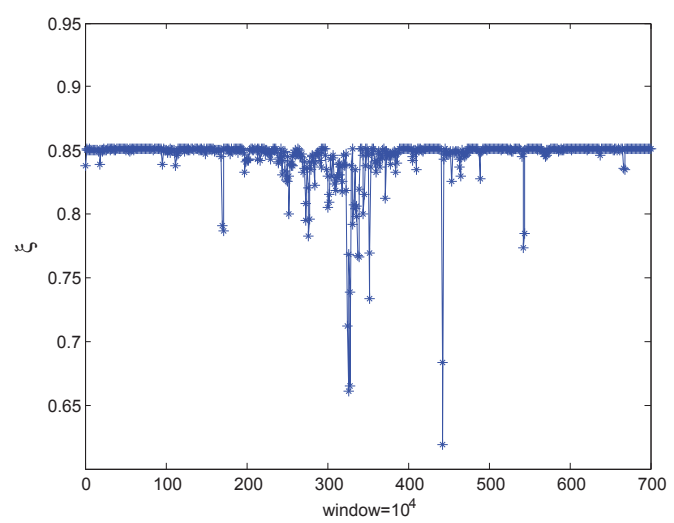

(a) The link of the right hip $\rightarrow$ the right ankle.

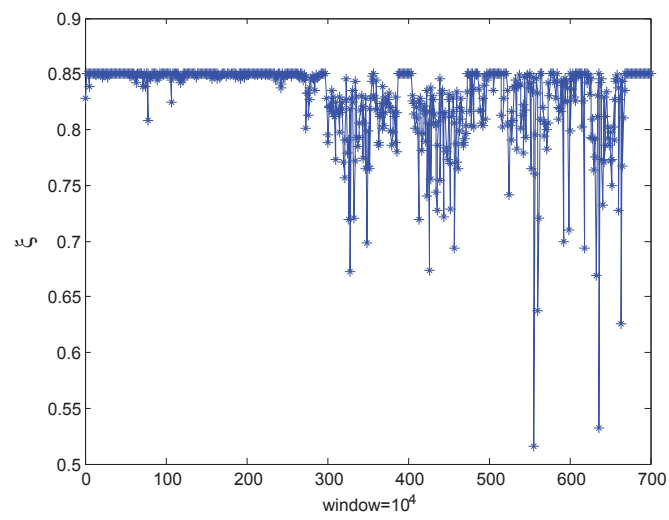

(b) The link of the right hip $\rightarrow$ the right wrist.

Fig. 2. The energy saving efficiency of link adaptation over power control.

PER introduced by LA and PC comparing with the static scheme. Figure 4 shows the empirical cumulative distribution function of the increased PER owing to PC. This figure can also represent the increased PER by LA, as the PER of LA is nearly the same as that of PC. It shows that this simple PC algorithm introduces below $1 \%$ additional packet erasures during $80-95 \%$ time at different links. In the worst case this algorithm introduces at most $5 \%$ additional packet erasures. Of course, there are other work such as [6], [7], [13] focusing on designing advanced algorithms to optimize the reliability and energy saving. It can be expected that the increased PER will be smaller if applying more advanced LA or PC algorithms.

\section{CONCLUSION}

This paper compares the energy saving efficiency of link adaptation and power control in BANs based on a generic energy consumption model. It sheds a light on how the average current at the transmitter and receiver, and the average data rate determine the energy saving efficiency of LA and PC. The condition that LA outperforms PC is also derived. Furthermore, to evaluate the energy saving efficiency, the simulation has applied the simple PC and LA algorithms to a large dataset measured in a BAN testbed. The simulation results show that LA is more efficient to save energy in BANs 


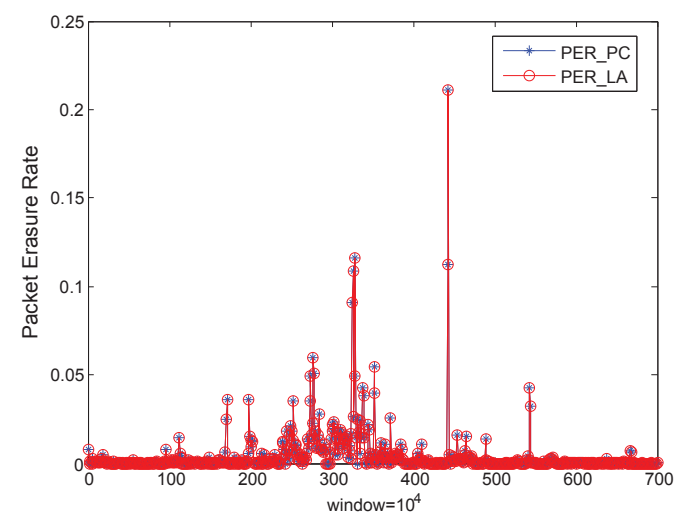

(a) The link of the right hip $\rightarrow$ the right ankle.

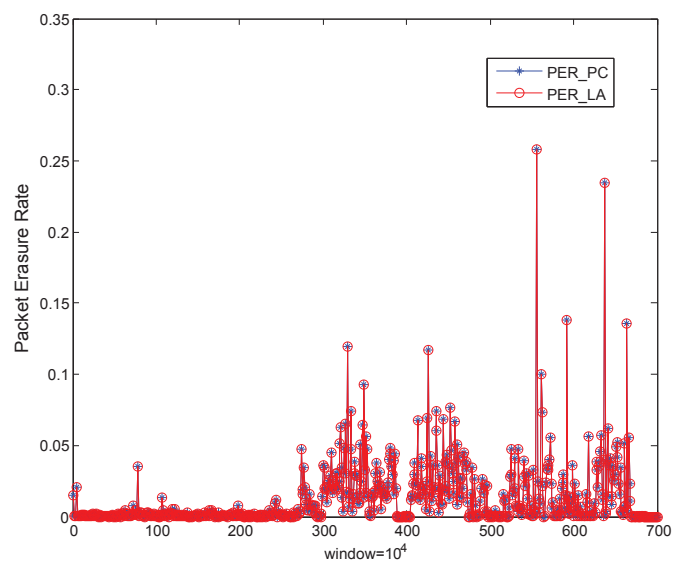

(b) The link of the right hip $\rightarrow$ the right wrist.

Fig. 3. Packet erasure rate of link adaptation and power control.

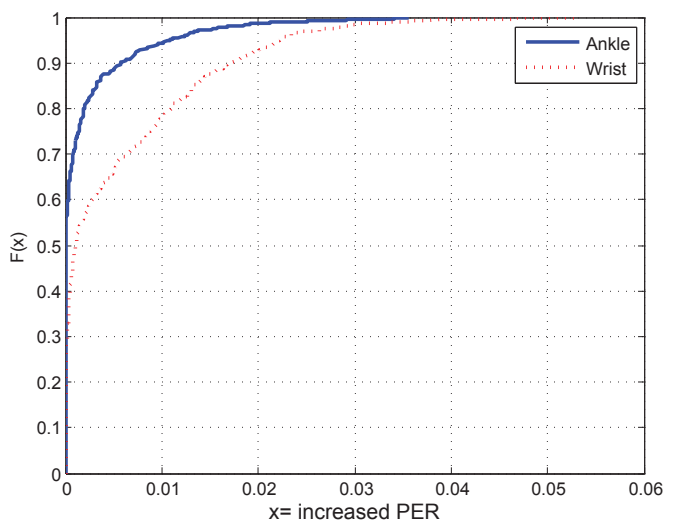

Fig. 4. The empirical cumulative distribution function of the increased PER owing to power control.

and it can save about $80-85 \%$ more energy than PC during $80 \%$ time. In terms of PER, LA and PC have similar performances. Comparing with the static scheme, the additional packet erasures introduced by the adaptive schemes is below $1 \%$ during $80-95 \%$ time.

\section{ACKNOWLEDGMENT}

The author would like to thank John Rohde and Qiang Li for helpful discussion on the transceiver electronics.

\section{REFERENCES}

[1] A. Boulis, D. Smith, D. Miniutti, L. Libman, and Y. Tselishchev, "Challenges in body area networks for healthcare: the mac," IEEE Communications Magazine, vol. 50, no. 5, pp. 100 -106, may 2012.

[2] E. Shih, S.-H. Cho, N. Ickes, R. Min, A. Sinha, A. Wang, and A. Chandrakasan, "Physical layer driven protocol and algorithm design for energy-efficient wireless sensor networks," in Proceedings of the 7th annual international conference on Mobile computing and networking, ser. MobiCom '01. ACM, 2001, pp. 272-287.

[3] D. Smith, L. Hanlen, J. Zhang, D. Miniutti, D. Rodda, and B. Gilbert, "Characterization of the dynamic narrowband on-body to off-body area channel," in IEEE International Conference on Communications, 2009. ICC '09, june 2009, pp. 1 -6.

[4] R. D'Errico and L. Ouvry, "Time-variant ban channel characterization," in Personal, Indoor and Mobile Radio Communications, 2009 IEEE 20th International Symposium on, sept. 2009, pp. 3000 -3004.

[5] S. Xiao, A. Dhamdhere, V. Sivaraman, and A. Burdett, "Transmission power control in body area sensor networks for healthcare monitoring," IEEE Journal on Selected Areas in Communications, vol. 27, no. 1, pp. $37-48$, january 2009.

[6] M. Quwaider, J. Rao, and S. Biswas, "Body-posture-based dynamic link power control in wearable sensor networks," IEEE Communications Magazine, vol. 48, no. 7, pp. $134-142$, july 2010.

[7] D. Smith, L. Hanlen, and D. Miniutti, "Transmit power control for wireless body area networks using novel channel prediction," in IEEE Wireless Communications and Networking Conference (WCNC), 2012, april 2012, pp. $684-688$.

[8] S. Lanzisera, A. Mehta, and K. Pister, "Reducing average power in wireless sensor networks through data rate adaptation," in IEEE International Conference on Communications, 2009. ICC '09., june 2009, pp. 1 -6.

[9] L. Hanlen, V. Chaganti, B. Gilbert, D. Rodda, T. Lamahewa, and D. Smith, "Open-source testbed for body area networks: $200 \mathrm{sample} / \mathrm{sec}$, 12 hrs continuous measurement," in 2010 IEEE 21st International Symposium on Personal, Indoor and Mobile Radio Communications Workshops (PIMRC Workshops),, sept. 2010, pp. $66-71$.

[10] A. Wang and C. Sodini, "A simple energy model for wireless microsensor transceivers," in IEEE Global Telecommunications Conference, 2004 GLOBECOM '04., vol. 5, nov.-3 dec. 2004, pp. 3205 - 3209 Vol.5.

[11] _ - "On the energy efficiency of wireless transceivers," in IEEE International Conference on Communications, 2006. ICC '06., vol. 8, june 2006, pp. $3783-3788$.

[12] A. Wang, S. Cho, C. Sodini, and A. Chandrakasan, "Energy efficient modulation and mac for asymmetric rf microsensor systems," in International Symposium on Low Power Electronics and Design, 2001., 2001, pp. $106-111$.

[13] F. Martelli, R. Verdone, and C. Buratti, "Link adaptation in wireless body area networks," in Vehicular Technology Conference (VTC Spring), 2011 IEEE 73rd, may 2011, pp. $1-5$.

[14] CC2420 data sheet: $2.4 \mathrm{GHz}$ IEEE 802.15.4 / ZigBee-ready RF Transceiver, Texas Instruments, 2010. [Online]. Available: http: //www.ti.com/lit/ds/symlink/cc2420.pdf

[15] "Ieee standard for local and metropolitan area networkspart 15.6: Wireless body area networks," IEEE Std 802.15.6-2012, pp. 1 -271, 292012.

[16] CC1000: Single Chip Very Low Power RF Transceiver, Texas Instruments, 2009. [Online]. Available: http://www.ti.com/lit/ds/symlink/ cc1000.pdf

[17] CC2520 data sheet: 2.4 GHZ IEEE 802.15.4/Zigbee RF Transceiver, Texas Instruments, 2007. [Online]. Available: http://www.ti.com/lit/ds/ symlink/cc2520.pdf

[18] L. Hanlen and D. Rodda, "Public dataset for ban radio channel models," March 2010, oneline: www.nicta.com.au/hpi.

[19] D. Smith, D. Miniutti, L. Hanlen, D. Rodda, and B. Gilbert, "Dynamic narrowband body area communications: Link-margin based performance analysis and second-order temporal statistics," in IEEE Wireless Communications and Networking Conference (WCNC), 2010, april 2010, pp. $1-6$. 